\title{
Contrast-associated acute kidney injury in elderly; a mini-review on nursing prospectives
}

\author{
Ali Hasanpour Dehkordi ${ }^{\circledR}$, Sam Mirfendereski $^{2^{*}}{ }^{\circledR}$, Abdolmajid Taheri $^{3^{\circledR}}$ \\ ${ }^{1}$ Social Determinants of Health, Research Center, School of Allied Medical Sciences, Shahrekord University of Medical Sciences, \\ Shahrekord, Iran \\ ${ }^{2}$ Department of Radiology, Isfahan University of Medical Sciences, Isfahan, Iran \\ ${ }^{3}$ Department of Radiology, Shahrekord University of Medical Sciences, Shahrekord, Iran
}

\section{A R T I C L E IN F O}

Article Type:

Mini Review

\section{Article History:}

Received: 23 April 2020

Accepted: 7 June 2020

Published online: 19 June 2020

\section{Keywords:}

Contrast-associated acute

kidney injury, Elderly, Contrast-

medium, Estimated glomerular

filtration rate

\begin{abstract}
A B S T R A C T
Contrast medium is used daily for accurate and effective diagnostic procedures. They are often necessary to provide an exact diagnosis and are almost safe and effective when used correctly. However contrast media can sometimes be life-threatening especially in elderly, since the elderly suffer from several problems. Aging is frequently accompanied by chronic diseases like chronic renal failure, comorbidity, frailty and disability. Therefore, it is important to know how reactions to contrast agents manifest and how to manage them immediately. Decreased kidney functions are sometimes seen after intravenous contrast media injection therefore radiologists should monitor all stages of the contrast injection. Accordingly, the medical team should have information on the complications, prevention and care of the patients under contrast media.
\end{abstract}

Implication for health policy/practice/research/medical education:

Patients, especially the elderly who are referred for contrast procedure, should be evaluated by a medical team prior to the procedure. Use of low- or iso osmolar contrast and avoidance of nephrotoxic agents such as nonsteroidal anti-inflammatory drug is suggested. Metformin should be discontinued before and 48 hours after contrast injection.

Please cite this paper as: Hasanpour Dehkordi A, Mirfendereski S, Taheri A. Contrast-associated acute kidney injury in elderly; a mini-review on nursing prospectives. J Renal Inj Prev. 2021; 10(1): e01. doi: 10.34172/jrip.2021.01.

\section{Introduction}

Chronic disease is one of the main causes of hospitalization and leads to additional costs for patients and administration. Over the past two decades, the elderly population has more than doubled (1-3). Aging is frequently accompanied by chronic diseases like chronic renal failure, comorbidity, frailty, and disability (1). As life expectancy increases, diagnostic procedures, such as MRI and CT scan, are also increasing. Since the elderly may have several chronic diseases at the same time, such as diabetes or kidney failure or they may take various medications; therefore, they are at greater risk of complications (4). While contrast agents are excreted through the kidneys and the glomerular filtration rate (GFR) is reduced in the elderly, paying attention to this aspect of elderly is of particular importance (1). Different types of contrast agents are used to improve medical images $(5,6)$. Contrast agents have side effects $(7)$. The undesirable side effects of contrast agents prescription vary from minor physiological disorders to severe and rare life-threatening risks $(6,7)$, therefore full familiarity with the emergency manifestation and treatment of contrast agents reactions should be considered especially in an environment where contrast agents are injected intravascularly (5-7). Recent studies showed annually, half of the approximately 76 million CTs and 34 million MRIs are performed through intravenous contrast agent injection (5,8), while contrast agents reduce kidney function, especially in high-risk patients containing elderly. With the increasing administration of contrast agents in diagnostic and therapeutic interventions and because of the high age of patients and the presence of 
comorbidities (6), kidney disorders caused by injection of contrast agents have become one of the most common causes of acute renal failure in this age population $(6,7,9)$. In fact, some of the kidney injuries caused by contrast agent are significant that lead to frequent hospitalizations, increased financial burden and increased mortality up to $20 \%$ (10). In fact, the third reason for hospitalization in acute renal failure patients is due to the use of contrast agents in cardiac angiography $(6,11)$. Nephropathy caused by contrast agents is one of the known complications following coronary artery catheterization. Its incidence is less than $3 \%$ in normal individuals however in highrisk patients such as those with kidney failure or diabetic patients reaches up to $50 \%(10,12)$. Although contrast agent-induced nephropathy is most likely to be reversible $(10,13,14)$, sometimes these people need dialysis and this can lead to an increased hospital stay, an increased medical cost, and increased hospital mortality of 12 to $37 \%$. There are several risk factors for contrast-induced nephropathy that diabetes is one of the most important and most common of them particularly in aging population $(10,11)$. Ideally, the contrast agent should be removed from the body without any complications $(5,8)$. According to what mentioned above, having modern equipment, skillful specialists and knowledge of emergency manifestation and treatment of contrast material reactions is a necessary entity. Some important guidelines are discussed here $(5,8,15)$.

Before injecting contrast agent, the following issues must be considered:

- Food and drug allergic reaction history, bronchospasm, heart and kidney diseases, especially in high-risk individuals such as the elderly over 60 years old and under 5 years old $(5,8,11,13,15)$.

- In the history of kidney disease, previous chronic kidney disease, transplantation and tumor, and a family history of kidney failure, insulin-treated diabetes, antihypertensive drugs and use of nephrotoxic drugs should be regarded.

- Estimated GFR (eGFR) $<30 \mathrm{~mL} / \mathrm{min} / 1.73 \mathrm{~m}^{2}$ before IV (intravenous) or IA (intraarterial) contrastmedium administration

- $\quad$ eGFR $<45 \mathrm{~mL} / \mathrm{min} / 1.73 \mathrm{~m}^{2}$ if the patient admitted on ICU or if intra-arterial

- Contrast-medium administration

- Presence of risk factors for impaired renal function in general (not specific for contrast-associated acute kidney injury; contrast-associated acute kidney injury), like hypercalcemia or presence of multiple myeloma

- Female gender

- Low body mass index

- Cardiovascular and metabolic risk factors

- Malignancy

- Inflammation
- $\quad$ Bleeding

- Anemia

- Hyperuricemia

- Procedure-related

- Repeated contrast-medium injections in a short period (48-72 hours)

- Administration of high doses of contrast-medium

- Ratio of contrast-medium dose to absolute eGFR should be $<1.1$

- Ratio of contrast-medium volume to eGFR should be $<3.0$ (if contrast-medium concentration is $350 \mathrm{mg}$ iodine/mL) $(5,6,8,11,16)$.

Side effects of contrast agents

The rout of contrast media administration can affect the side effects. In oral/rectal use, these effects are reduced because absorption in the gastrointestinal tract is very low.

Side effects of this method are heartburn, abdominal pain and transient diarrhea especially if their volume is high and high in concentration.

Thesideeffectsofintra-articularinjection (arthrography) are pain and swelling. The most common side effects of contrast media related to intravenous injection include topical and systemic reactions like an anaphylactic shock that is life-threatening, nausea, vomiting, a metallic taste in the mouth, and generalized warmth or flushing, and renal failure between $1 \%$ and $13 \%$ of cases, proteinuria, damage to the tissue and compartment syndrome due to contrast agent leakage $(6,12,17)$.

Treatment of side effects Flushing and feeling of warmth

There is no need for special treatment (if the patient has stress, these side effects would be exacerbated, talk about harmless things to avoid stress).

\section{Skin side effects}

For skin side effects such as severe hives and redness intravenous hydrocortisone injection is suggested. A doctor must do this procedure.

Nausea and restlessness

Deep breathing by the patient, breathing out, relaxing the chest and visit by the physician $(8,18-20)$.

Treatment of shock symptoms

Shock symptoms: Skin pallor, sweating, rapid pulse

The treatment: Trendelenburg position, notifying the physician.

What should be done for patients with rapid and very weak breathing, loss of consciousness or cardiac arrest? Call the physician, cardiac-pulmonary resuscitation, Trendelenburg position, using airway, using oxygen mask, (capsule should be filled), pulse control, blood pressure 
control (put a pressure gauge on the arm).

\section{High risk patients}

High-risk patients are those with history of contrast agent reaction, older patients, heart disease, diabetic patients, the existence of disorder in the blood-brain barrier, patients with renal failure, patients with radiographic examinations who need high doses $(8,18-20)$.

Prevention of complications in patients under contrast media include

IV hydration with normal saline or oral hydration, minimizing contrast volume, use of sodium bicarbonate (21), N-acetylcysteine (NAC), calcium channel blockers, adenosine antagonists, prostaglandin analogs, L-arginine, statins, atrial natriuretic peptide, endothelin antagonists, dopamine, fenoldopam, hypertonic mannitol and furosemide (7,22-24). Discontinuation of metformin 48 hours after contrast injection.

\section{Discussion}

Contrast-induced reactions including nephrotoxicity are serious side effects that require preventive care because treatment options are of limited value. Physicians using contrast media should incorporate prevention strategies into their clinical practice. Consultant nephrologists can help radiologists and cardiologists identify high-risk patients and recommend the best practice to reduce the incidence of contrast-associated acute kidney injury (25). Recent studies show that contrast media is increasingly used for diagnostic procedures in the elderly (26). In the elderly, anatomical and physiological changes in the kidneys, due to the process of aging, increases the susceptibility of kidneys to structural dysfunction (26). As the elderly are more at risk than other age groups there is a need for preventive protocols before and after contrastenhanced examinations in the elderly.

\section{Conclusion}

Patients, especially the elderly who are referred for contrast procedure, should be evaluated by a medical team prior to the procedure since care should be given before and after contrast injection. Use of low- or iso osmolar contrast and avoidance of nephrotoxic agents such as nonsteroidal anti-inflammatory drug is suggested. Metformin should be discontinued before and up to 48 hours after contrast injection. Additionally, it is recommended that the contrast media be avoided or used at the lowest possible volume and accordingly regular monitor of renal function tests in high risk patients should also be considered.

\section{Authors' contribution}

AHD searched the literature, prepared the manuscript and edited the paper. Other authors contributed equally to this study. All authors critically revised and approved the final manuscript

Conflicts of interest

The authors declared no competing interests.

\section{Ethical considerations}

Ethical issues (including plagiarism, data fabrication, double publication) have been completely observed by the authors.

\section{Funding/Support}

None.

\section{References}

1. Mallappallil M, Friedman EA, Delano BG, McFarlane SI, Salifu MO. Chronic kidney disease in the elderly: evaluation and management. Clin Pract (Lond) 2014;11(5):525-535. doi:10.2217/cpr.14.46.

2. Stevens LA, Viswanathan G, Weiner DE. Chronic kidney disease and end-stage renal disease in the elderly population: current prevalence, future projections, and clinical significance. Adv Chronic Kidney Dis 2010;17 (4):293-301. doi:10.1053/j.ackd.2010.03.010.

3. Blagg CR. The early history of dialysis for chronic renal failure in the United States: a view from Seattle. Am J Kidney Dis 2007;49 (3):482-496. doi:10.1053/j.ajkd.2007.01.017.

4. Nobili A, Garattini S, Mannucci PM. Multiple diseases and polypharmacy in the elderly: challenges for the internist of the third millennium. J Comorb. 2011;1:28-44. Published 2011 Dec 27. doi:10.15256/joc.2011.1.4

5. Beckett KR, Moriarity AK, Langer JM. Safe use of contrast media: what the radiologist needs to know. Radiographics 2015;35(6):1738-50.doi: 10.1148/rg.2015150033.

6. Vandenberghe W, Hoste E. Contrast-associated acute kidney injury: does it really exist, and if so, what to do about it? Version 1. F1000Res. 2019; 8: F1000 Faculty Rev-753. doi: 10.12688/f1000research.16347.1.

7. Seeliger E, Sendeski M, Rihal CS, Persson PB. Contrastinduced kidney injury: mechanisms, risk factors, and prevention. Eur Heart J. 2012;33(16):2007-2015. doi:10.1093/eurheartj/ehr494

8. Brenner DJ, Hricak H. Radiation exposure from medical imaging: time to regulate? JAMA 2010;304(2):208-9.doi: 10.1001/jama.2010.973.

9. Liu Y, Liang X, Xin S, Liu J, Sun G, Chen S, et al. Risk factors for contrast-induced acute kidney injury (CI-AKI): protocol for systematic review and meta-analysis. BMJ -Open 2019;9(8):e030048. doi: 10.1136/bmjopen-2019-030048.

10. Geenen RW, Kingma HJ, van der Molen AJ. Contrastinduced nephropathy: pharmacology, pathophysiology and prevention. Insights Imaging. 2013;4(6):811-820. doi:10.1007/s13244-013-0291-3

11. Gruberg L, Mehran R, Dangas G, Mintz GS, Waksman R, Kent KM, et al. Acute renal failure requiring dialysis after percutaneous coronary interventions. Catheter cardiovasc Interv 2001;52(4):409-16.doi: 10.1002/ccd.1093.

12. Bartorelli AL, Marenzi G. Contrast-induced nephropathy. J Interv Cardiol 2008;21(1):74-85.

13. Goldenberg I, Matetzky S. Nephropathy induced by contrast media: pathogenesis, risk factors and preventive 
strategies [published correction appears in CMAJ. 2005 Nov 8;173(10):1210]. CMAJ. 2005;172(11):1461-1471. doi: 10.1503/cmaj.1040847.

14. Mehran R, Aymong ED, Nikolsky E, Lasic Z, Iakovou I, Fahy M, et al. A simple risk score for prediction of contrast-induced nephropathy after percutaneous coronary intervention: development and initial validation. J Am Coll Cardiol. 2004;44:1393-9. doi: 10.1016/j.jacc.2004.06.068.

15. van der Molen AJ, Reimer P, Dekkers IA, Bongartz G, Bellin M-F, Bertolotto M, et al. Post-contrast acute kidney injury. Part 2: risk stratification, role of hydration and other prophylactic measures, patients taking metformin andchronic dialysis patients. Eur Radiol 2018;28:2856-69. doi: 10.1007/s00330-017-5247-4.

16. Stacul F, van der Molen AJ, Reimer P, Webb JA, Thomsen HS, Morcos SK, et al. Contrast induced nephropathy: updated ESUR contrast media safety committee guidelines. Eur Radiol. 2011;12:41-2527.

17. Caschera L, Lazzara A, Piergallini L, Ricci D, Tuscano B, Vanzulli A. Contrast agents in diagnostic imaging: present and future. Pharmacol Res. 2016;110:65-75. doi: 10.1016/j. phrs.2016.04.023.

18. Bashir MR, Bhatti L, Marin D, Nelson RC. Emerging applications for ferumoxytol as a contrast agent in MRI. J Magn Reson Imaging. 2015;41:884-98. doi: 10.1002/ jmri.24691.

19. Louza J, Krause E, Gurkov R. Hearing function after intratympanic application of gadolinium-based contrast agent: a long-term evaluation. Laryngoscope 2015;125:236670. doi: 10.1002/lary.25259.

20. Piskunowicz M, Kosiak W, Batko T, Piankowski A,
Połczynska K, Adamkiewicz-Drozynska E. Safety of intravenous application of second-generation ultrasound contrast agent in children: prospective analysis. Ultrasound Med Biol. 2015;41:1095-9. doi: 10.1016/j. ultrasmedbio.2014.11.003.

21. Gurm HS, Dixon SR, Smith DE, Share D, LaLonde T, Greenbaum A, et al. Renal function-based contrast dosing to define safe limits of radiographic contrast media in patients undergoing percutaneous coronary interventions. J Am Coll Cardiol. 2011;58:907-14. doi: 10.1016/j. jacc.2011.05.023.

22. Brar SS, Aharonian V, Mansukhani P, Moore N, Shen AY, Jorgensen $M$, et al. Haemodynamic-guided fluid administration for the prevention of contrast-induced acute kidney injury: the POSEIDON randomised controlled trial. Lancet 2014;383:1814-23. doi:10.1016/j.jvs.2014.09.019.

23. Feldkamp T, Kribben A. Contrast media induced nephropathy: definition, incidence, outcome, pathophysiology, risk factors and prevention. Minerva Medica. 2008;99:177-96.

24. Briguori C, Marenzi G. Contrast-induced nephropathy: pharmacological prophylaxis. Kidney Int Suppl. 2006;69:S30-8. doi: 10.1038/sj.ki.5000372.

25. Ludwig U, Keller F. Prophylaxis of contrast-induced Nephrotoxicity. Biomed Res Int. 2014;2014:308316. doi: $10.1155 / 2014 / 308316$.

26. Aoki BB, Fram D, Taminato M, Batista RES, Belasco A, Barbosa DA. Acute kidney injury after contrast-enhanced examination among elderly. Rev Lat Am Enfermagem. 2014;22:637-44. doi: 10.1590/0104-1169.3440.2462.

Copyright ( $) 2021$ The Author(s); Published by Nickan Research Institute. This is an open-access article distributed under the terms of the Creative Commons Attribution License (http://creativecommons.org/licenses/by/4.0), which permits unrestricted use, distribution, and reproduction in any medium, provided the original work is properly cited. 